\title{
Sobrecarga del cuidador de adultos mayores dependientes
}

\author{
Chango M. Viviana. N. \\ vchango8686@uta.edu.ec \\ https://orcid.org/0000-0002-8357-5912 \\ Facultad Ciencias de la Salud, Estudiante de la Carrera de Enfermería, \\ Universidad Técnica de Ambato \\ Ambato-Ecuador. \\ Guarate C. Yeisy C. \\ yc.guarate@uta.edu.ec \\ https://orcid.org/0000-0003-1526-4693 \\ Licenciada en Enfermería, \\ Magister en Enfermería en Salud Reproductiva, \\ Dra. En Enfermería en Salud y Cuidado Humano, \\ Universidad de Carabobo, Venezuela. \\ Docente de la Carrera de Enfermería, \\ Universidad Técnica de Ambato, Ecuador.
}

\section{RESUMEN}

Introducción: El envejecimiento es un proceso dinámico, progresivo e irreversible que da lugar a múltiples cambios afectando la capacidad de realizar las actividades diarias por cuenta propia, por lo tanto, los adultos mayores requieren de un cuidador, es decir, una persona que le dispense el cuidado sobre todo si presenta discapacidad o dependencia. Objetivo: Determinar la prevalencia de la sobrecarga del cuidador de adultos mayores dependientes. Metodología: Estudio cuantitativo, descriptivo, de corte transversal y de campo. La población estuvo conformada por 90 cuidadores primarios de una población adulta mayor a quienes se les aplicó el test de Zarit previa firma de consentimiento informado; siendo la muestra censal. Para la recolección de la información se tomó en cuenta los señalado en la Declaración de Helsinki relacionado con los aspectos éticos para la investigación con seres humanos. Resultados: El mayor porcentaje de los cuidadores eran se sexo femenino quienes presentaron niveles de sobrecarga intensa. Conclusión: Existe una sobrecarga intensa en la población de estudio por lo que es importante la intervención del personal de salud para evitar que esta sobrecarga afecte la vida del cuidador y por ende la del adulto mayor.

Palabras claves: sobrecarga; cuidadores; adulto mayor 


\title{
Overload of the dependent elderly caregiver
}

\begin{abstract}
Introduction: Aging is a dynamic, progressive and irreversible process that results in multiple changes affecting the ability to perform daily activities on their own, therefore, older adults require a caregiver, that is, a person who dispenses care especially if they present disability or dependence. Objective: To determine the prevalence of caregiver overload in dependent older adults. Methodology: Quantitative, descriptive, crosssectional and field study. The population consisted of 90 primary caregivers of an older adult population to whom the Zarit test was applied after signing an informed consent form; being the census sample. For the collection of the information, the Declaration of Helsinki related to the ethical aspects of research with human beings was taken into account. Results: The highest percentage of caregivers were women who presented levels of intense overload. Conclusion: There is an intense overload in the study population, so it is important for health personnel to intervene to prevent this overload from affecting the caregiver's life and, therefore, that of the older adult.
\end{abstract}

Key words: overload; caregivers; elderly.

Artículo recibido: 02 noviembre. 2021 Aceptado para publicación: 28 noviembre 2021 Correspondencia: vchango8686@uta.edu.ec Conflictos de Interés: Ninguna que declarar 


\section{INTRODUCION}

El envejecimiento se define como un proceso dinámico, progresivo e irreversible dando lugar a múltiples cambios biológicos, psicológicos y sociales; el ciclo de vida inicia con la concepción, se desarrolla durante toda vida y culmina con la muerte (OMS, 2021)

El mundo experimenta un cambio demográfico, estimando que para el 2050 la población mundial en la edad de 60 años llegue a los 2000 millones, un aumento de 900 millones con respecto a 2015. Hoy en día hay 125 millones de personas con 80 años o más. Para 2050 se estima un número casi igual de personas en este rango de edad solamente en China y 434 millones de personas en todo el mundo. Para 2050, aproximadamente un $80 \%$ de todas las personas mayores vivirían en países de ingresos bajos y medianos. La pauta de envejecimiento de la población en todo el mundo aumenta rápidamente. En Francia se dispuso de casi 150 años para adaptarse a un incremento del $10 \%$ al $20 \%$ en la población mayor de 60 años. En Canadá el 9,6\% de la población brinda ayuda a personas que están siendo afectados por alguna enfermedad crónica. Dentro de la población norteamericana, el 19,2\%, es decir, 52 millones de personas cuidan de personas adultas enfermas (Rizo et al., 2016). De igual manera, en Ecuador existe un aproximado de 1.049.824 personas mayores de 65 años lo que representa el 6,5\% de la población total (MIES, 2021).

El envejecimiento es un área de estudio de magnitud, comorbilidad y vulnerabilidad social, en especial en los adultos mayores que atraviesan una situación de dependencia (Minsalud, 2021). Dentro de los adultos mayores están quienes se jubilaron y reciben pensiones que les permite vivir dignamente y que son de ayuda para poder solventar sus propios gastos siendo un gran apoyo económico para que su cuidador pueda costear algún tratamiento o medicamentos que necesite, sin embargo, también existen adultos mayores que no cuentan con este apoyo y sus familiares tienen la obligación de solventar los gastos económicos para poder darles una vida digna a la persona bajo su cargo, para este grupo de adultos mayores los años adicionales se acompañan del declive de sus capacidades física y mental y por ende las complicaciones son aún más notorias (Toro, 2016).

Como consecuencia del alargamiento de la vida también aumenta el número de enfermedades degenerativas y crónicas; a medida que la persona va envejeciendo se van alterando sus capacidades físicas y mentales afectando, de tal manera, la capacidad de 
realizar las actividades diarias por su propia cuenta, perjudicando la independencia de la persona (Zepeda, 2019).

El aumento de adultos mayores con algún tipo de discapacidad o de dependencia requiere la presencia de un cuidador, es decir, de una persona que realice los cuidados directos del adulto mayor dependiente. Esta persona, generalmente un cuidador informal, dispensa de manera altruista y gratuita el cuidado de una persona que presenta algún grado de discapacidad o dependencia, por lo general, la responsabilidad recae en un familiar directo que conviva con la persona (D’Argemir, 2015). Es importante resaltar que éste rol casi siempre es asumido por el sexo femenino quien no recibe algún tipo de ayuda o remuneración por los cuidados brindados, debe contar con una buena relación afectiva con la persona a su cargo, además debe poseer capacidades para ofrecerle seguridad y bienestar al adulto mayor (Silva et al., 2016).

Los cuidadores informales tienen la responsabilidad de realizar diferentes actividades como esfuerzos físicos viéndose sometidos a tensiones que derivan del propio cuidado conllevando a cambios en su ambiente familiar, laboral y social afectando así la calidad de vida del cuidador al no poder administrar su propio tiempo y descuidando su salud; esto lo hace vulnerable a sufrir de enfermedades (Gomez et al., 2016).

Así mismo, el cuidar de un adulto mayor que presente algún tipo de discapacidad o enfermedad crónica puede causar en el cuidador sentimiento de soledad, sobrecarga emocional, depresión, restricción de libertad personal, tiempo de ocio y descanso generando problemas en su salud como hipertensión arterial, problemas cardiovasculares y gastrointestinales, cefalea y alteraciones osteomusculares (Eterovic, 2015). La sobrecarga del cuidador aumenta con el nivel de dependencia de la persona cuidada, influyendo negativamente en la calidad de vida y en el estado psicológico de los adultos mayores dependientes y sobre todo en los cuidadores; es por esta razón que la sobrecarga de los cuidadores también se ha convertido hoy en día como un problema sanitario y social que requiere de más estudios para poder brindarle apoyo y soluciones a través de intervenciones por parte de un profesional en Psicología y otros miembros de la salud (Gonzales, L et al., 2016).

Es por ello que esta investigación tuvo como objetivo general determinar la prevalencia de la sobrecarga del cuidador de adultos mayores dependientes, mediante la intervención de profesionales de la salud a través de interconsultas con psicología y educación por 
parte de enfermería para poder disminuir la sobrecarga existente y así evitar la despersonalización y complicaciones en el cuidador.

\section{ESTRATEGIAS METODOLÓGICAS O MATERIALES Y MÉTODOS}

El estudio realizado es de enfoque cuantitativo, descriptivo, de corte transversal y de campo (Gavira, 2015), lo cual permitió determinar la prevalencia de la sobrecarga del cuidador de adultos mayores dependientes. La población estuvo conformada por 90 cuidadores primarios de la población adulta mayor del Cantón Cevallos de la provincia de Tungurahua. La muestra fue censal lo que quiere decir que se ocupó el $100 \%$ de la población. Para la recolección de la información se aplicó el test de Zarit, validado en varios idiomas incluido el español, además tiene una confiabilidad de 0.91 según el alfa de crombach (Rodriguez et al., 2016). Este test consta de 22 ítems con respuestas donde se evalúan de acuerdo a una escala de Likert que va de 1 (nunca) a 5 (casi siempre), donde el punto de corte es de 46 puntos que es indicativo de no sobrecarga; de 47-55 hay una sobrecarga leve y mayor a 56 puntos implica una sobre carga intensa (Tartaglini, 2019), por consiguiente, los resultados que se reflejarán en la evaluación indicarán las repercusiones negativas en el cuidador principal (Buritica et al., 2020) al prestar cuidados en el adulto mayor, como su salud física y mental, vida social y los recursos económicos (Navarro et al., 2017).

Para el procesamiento de la información se utilizó el programa Microsoft Excel para calcular la frecuencia y los porcentajes de los 22 ítems del test y las opciones de respuesta de la escala de Likert. De esta manera se pudieron ordenar los datos obtenidos y presentarlos en gráficas de barras (Quispe et al., 2019).

La investigación siguió los lineamientos de la declaración de Helsinki por lo cual los participantes fueron informados del propósito del estudio solicitando la firma de consentimiento informado previo al llenado del test. Así mismo, se cuidaron los principios de respeto, autonomía y confidencialidad de los participantes. (Chavez et al., 2018).

\section{RESULTADOS Y DISCUSIÓN}

\section{Resultados}

El gráfico 1 señala que, de 90 cuidadores de adultos mayores dependientes, el mayor porcentaje de cuidadores corresponde al sexo femenino con el $69 \%$, mientras que el $31 \%$ de los cuidadores son de sexo masculino. 
Gráfico 1: Distribución de la población según sexo

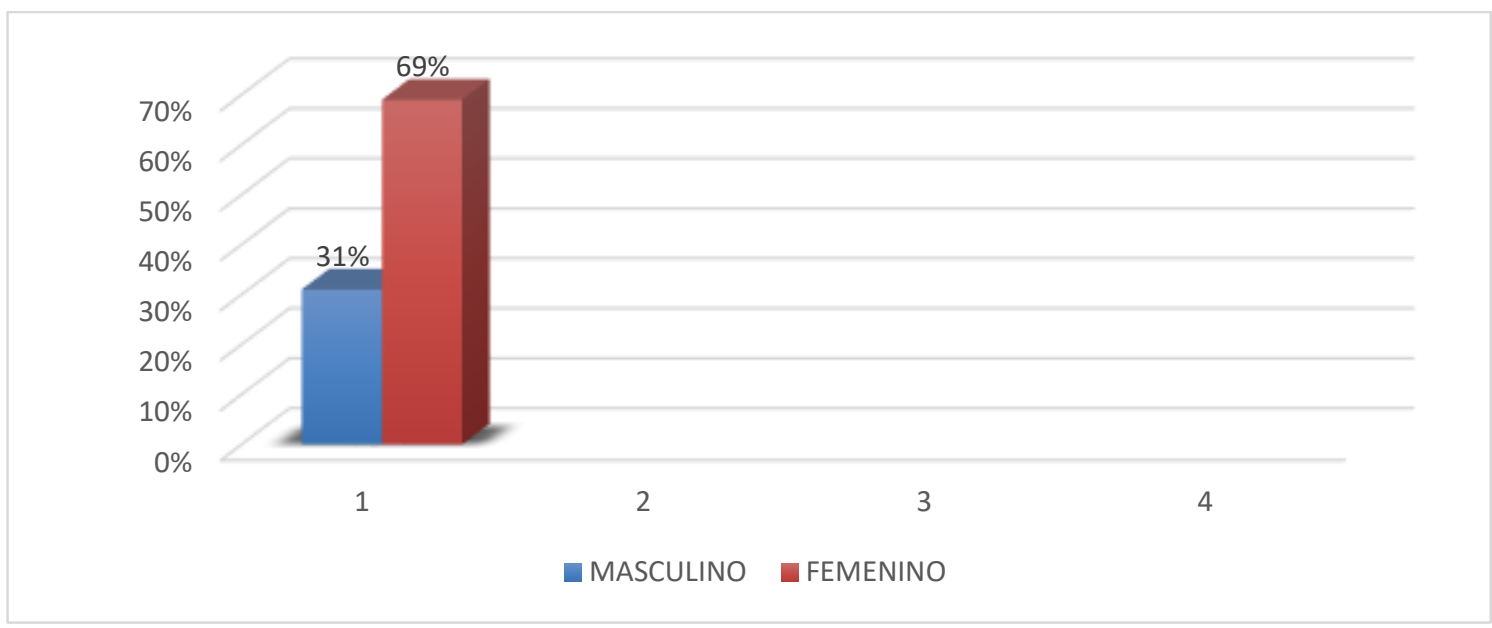

Fuente: Instrumento aplicado

En el gráfico 2 se evidencia que el 78\% de la población muestra una sobrecarga intensa, mientras que el $11 \%$ de la población señala que presenta una sobrecarga ligera, finalmente el $11 \%$ de los cuidadores de adultos mayores se encuentran con ausencia de sobrecargo.

Gráfico 2. Resultados del test de Zarit con relación al nivel de sobrecarga

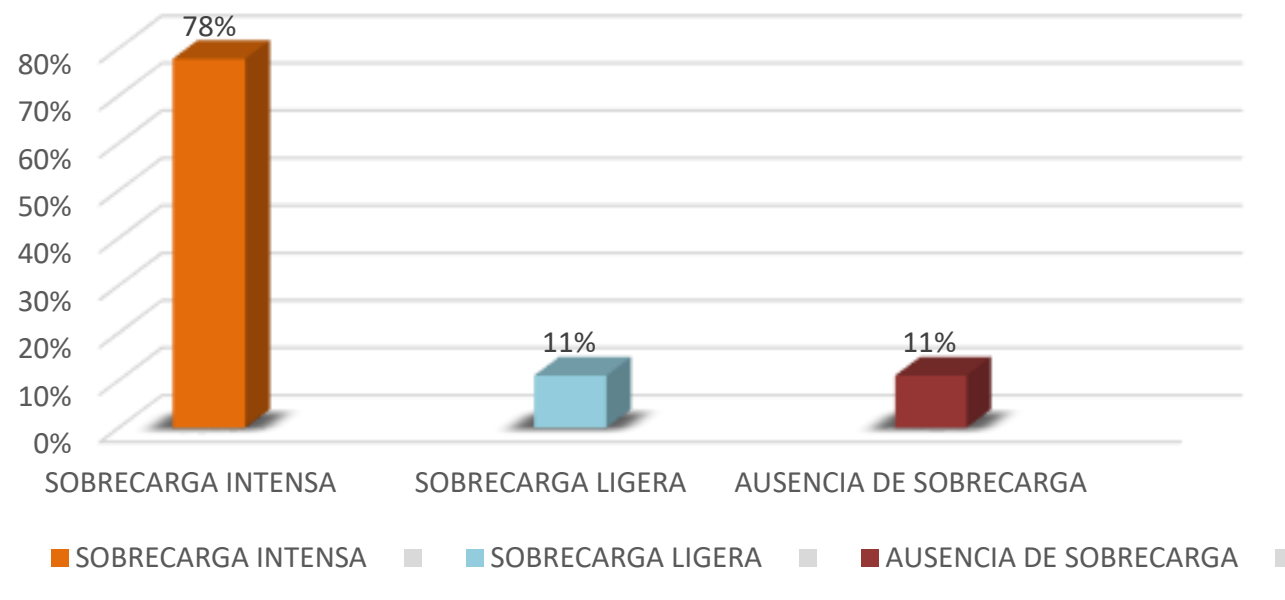

Fuente: Instrumento aplicado

\section{Discusión}

Los resultados del estudio evidenciaron que la población de estudio presentó una sobrecarga intensa coincidiendo con los hallazgos de una investigación realizada en Chile en donde se evidenció que el 59,7\% presentó una sobre carga intensa, mientras que el 23\%, una sobrecarga ligera (Flores et al., 2012). En una investigación realizada en Chile por Toro Yeny. Rivas Edith (2016) el 75,9\% de los cuidadores estudiados presentaron 
sobrecarga y el 24,1\% está libre de sobrecarga (Toro, Y et al., 2016). Resultados diferentes se halló en una investigación realizada en Lima, Perú señala que los cuidadores presentan un 11,5\% sobrecarga ligera (Rizo et al., 2016).

Por tal motivo, la sobrecarga del cuidador origina cambios relacionados con la familia, el trabajo, en lo económico, el tiempo de ocio, el estado de ánimo y el estado de salud, generando en el cuidador una serie de malestar tanto físico como mental. Esta serie de malestares no son tomados en cuenta por los cuidadores por el hecho de tratar de brindarles todos los cuidados necesarios al adulto mayor, haciendo de lado estas señales y no cuidar de la salud del cuidador (Delgado, E et al., 2016).

A través de esta investigación también se pudo evidenciar que la mayoría de los cuidadores eran de sexo femenino, lo que coincide con otras investigaciones realizada en México y Perú donde el 72.4\% y 86.9\% de la población, respectivamente, eran mujeres quienes además de tener responsabilidades en su hogar asumían el cuidado de un adulto mayor (Navarro et al., 2017). (Silva et al., 2016). Concluyendo que las mujeres son quienes asumen esta responsabilidad de cuidadores de adultos mayores mientras que los hombres asumen el rol de cuidador solo cuando no existe una mujer en su círculo familiar (Swinkels, J et al., 2019).

Lo antes expuesto señala que es la mujer quien asume el papel principal de cuidador principal, quien no solo se hace responsable de las tareas domésticas también asume el cuidado del adulto mayor y realiza actividades de cuidado de su propia salud familiar (Mosquera, I et al., 2019). Estudios realizados muestran que las mujeres que asumen el cargo de cuidadora principal o informal presentan casi dos veces más sobrecarga que los hombres y las cuidadoras más jóvenes presentan mayor sobrecarga que las de mayor edad por tratar de compatibilizar el empleo, las actividades sociales y el desarrollo profesional (Zepeda et al., 2019).

Por lo tanto, debido al envejecimiento de la población y al incremento de las enfermedades crónico degenerativas se ve la necesidad de contar con cuidadores responsables que puedan brindar los cuidados indispensables al adulto mayor sin descuidar su propia salud y en beneficio de las personas a su cargo (Cascella, $\mathrm{G}$ et al., 2020). 


\section{CONCLUSIONES}

El cuidar de un adulto mayor no debería depender de una sola persona, sino más bien debería estar a cargo de todos los miembros de la familia, para que de esta manera puedan evitarse una sobrecarga intensa ya que esta sobrecarga puede traer un costo muy alto en la vida de la única persona cuidadora, es por ello que se debería compartir y organizar el trabajo con los demás miembros de la familia de forma equitativa e igualitaria.

Los cuidadores presentan una sobrecarga intensa, por lo que necesita de ayuda por parte del personal de salud para evitar que esta sobrecarga afecte su vida, tanto en su salud como la su vida personal.

Generalmente los cuidadores de adultos mayores son de sexo femenino por lo que además de cumplir con este rol, desempeñan otras tareas como los quehaceres del hogar y encargarse de su propia familia, lo que podría afectar su salud física y mental, generando un estrés crónico, provocando cansancio emocional y también despersonalización al creer que no satisfacen las necesidades del adulto mayor bajo su cuidado por tener otras ocupaciones que cumplir.

Cuando un cuidador presenta sobrecarga tiende a abandonar algunas actividades; es por ello que para poder cuidar de la persona a su cargo empiezan a descuidar su vida social, por tal motivo, se recomienda al personal de salud intervenir implementando estrategias que apoyen a los cuidadores, con la finalidad de poder disminuir la sobrecarga para que el cuidador pueda tener una mejor calidad de vida y su estado de salud no se vea afectada. La premisa es cuidar al cuidador.

Agradecimientos: Agradezco a la Universidad Técnica de Ambato por el apoyo brindado con el respectivo trámite y, en especial, a la directora y a quienes conforman el Centro de Salud Tipo B Cevallos por permitirme llevar a cabo esta investigación

\section{LISTA DE REFERENCIAS}

Buritica, N., Buitrago, L., y Chaquir, MS. (2017). Burnout en cuidadores formales e informales del adulto mayor. revisión integrativa de la literatura. Cult Cuid, 17(1):80-92. Disponible: http://www.scielo.org.mx/pdf/eu/v12n1/v12n1a4.pdf

Cascella, G., y Garcia, R. (2020). Sobrecarga y desigualdad de género en el cuidado informal. Investigación y Educación en Enfermería, 38 (1), e10. Disponible en: http://www.scielo.org.co/scielo.php?pid=S0120$\underline{53072020000100010 \& \text { script }=\text { sci_arttext\&tlng=es\#B46 }}$ 
Chávez, N., y Estupiñán, S. (2018). Principios éticos rectores de las investigaciones de tercer nivel o grado académico en Ciencias Médicas. Revista Cubana de educación superior, 32(2). Disponible en: https://www.medigraphic.com/pdfs/educacion/cem-2018/cem182z.pdf

D’Argemir, D. (2015). Los cuidados de larga duración y el cuarto pilar del sistema de bienestar. Revista de Antropología Social, 24. DOI: $\underline{\text { https://doi.org/10.5209/rev_RASO.2015.v24.50663 }}$

Delgado, E., Barajas, T., Uriostegui, L., y López, V. (2016). Sobrecarga y percepción de la calidad de vida relacionada con el cuidador primario del paciente hemodializado. Revista Cubana de Medicina General Integral, 32(4), 1-9. Disponible en: http://scielo.sld.cu/scielo.php?script=sci_arttext\&pid=S0864$\underline{21252016000400006 \& \operatorname{lng}=\mathrm{es} \& \ln g=\mathrm{es}}$

Eterovic, C., Mendoza, S., y Sáez K. (2015). Habilidad de cuidado y nivel de sobrecarga en cuidadoras/es informales de personas dependientes. Enferm Glob. 2015;14(2):235.

Disponible

en:

https://scielo.isciii.es/scielo.php?script=sci_abstract\&pid=S1695$\underline{61412015000200013}$

Flores, E., Rivas, E., y Seguel F. (2012). Nivel de sobrecarga en el desempeño el rol del cuidador familiar de adulto mayor con dependencia severa. Cienc y Enferm. ;28(1):29-41.

Disponible

en:

https://www.scielo.cl/scielo.php?script=sci_arttext\&pid=S0717-

95532012000100004\&lng=en\&nrm=iso\&tlng=en

Gavira, S., y Osuna, J. (2015). La triangulación de datos como estrategia en investigación educativa. Pixel-Bit Rev Medios Educ;73-88. Disponible en: https://www.redalyc.org/articulo.oa?id=36841180005

Gómez, S., Ferrer, J., y Rigla F. (2016). La sobrecarga de las cuidadoras de personas dependientes: análisis y propuestas de intervención psicosocial. [Tesis de pregrado]:311. Disponible en: http://envejecimiento.csic.es/documentos/documentos/polibienestar-sobrecarga$\underline{02 . p d f}$

Gonzales, L., Real, J., Borrás, A., Martinez, J., Rodrigo, Virginia., y Navarro, D. (2016). Asociaciones entre cuidados informales, enfermedad y factores de riesgo: un 
estudio de ámbito nacional español. J Public Health Pol 37, 173-189. DOI: https://doi.org/10.1057/jphp.2016.3

MIES. (2021). Dirección Población Adulta Mayor. Disponible en: https://www.inclusion.gob.ec/direccion-poblacion-adulta-mayor/\#

Minsalud. (2021). Envejecimiento y Vejez. Disponible en: https://www.minsalud.gov.co/proteccionsocial/promocionsocial/Paginas/envejecimiento-vejez.aspx

Mosquera, I., Larrañaga, I., Lozada, M., Calderon, C., Machón, M., y Garcia, M. (2019). Desigualdades de género en los impactos del cuidado informal de mayores dependientes en Gipuzkoa: Estudio CUIDAR-SE. Rev. Esp. Salud publica 93, e201901002. Disponible en: https://www.scielosp.org/article/resp/2019.v93/e201901002/\#

Navarro, C., Uriostegui, L., Delgado, E., y Sahagún, N. (2017). Sobre la Depresión y sobrecarga en cuidadores primarios de pacientes geriátricos con dependencia física de la UMF 17. Revista Médica del Instituto Mexicano del Seguro Social;55(1). Disponible en: https://www.redalyc.org/articulo.oa?id=457749297013

OMS. (2021). Envejecimiento y salud. Disponible en: https://www.who.int/es/newsroom/fact-sheets/detail/envejecimiento-y-salud

Quispe, A., Calla, K., Yangali, J., Rodriguez, J., y Pumacayo, I. (2019). Estadística-noparamétrica-aplicada.pdf [Internet]. Disponible en: https://www.editorialeidec.com/wpcontent/uploads/2020/01/Estad\%C3\%ADstica-no-param\%C3\%A9tricaaplicada.pdf

Rizo, A., Marlén, L., Milián, N., Pagán, P., y Machado, J. (2016). Caracterización del cuidador primario de enfermo oncológico en estado avanzado. Rev Cubana Med Gen Integr. 32(3). Disponible en: http://scielo.sld.cu/scielo.php?script=sci_arttext\&pid=S0864$\underline{21252016000300005}$

Rodríguez, A., Cerquera, A., y Pabón, D. (2016). Escala de sobrecarga del cuidador Zarit: estructura factorial en cuidadores informales de Bucaramanga. Revista de 
Psicología Universidad de Antioquia. 8(2), 87-99. Disponible en: https://revistas.udea.edu.co/index.php/psicologia/article/view/327888

Silva, J., Gonzales, J., Mas, T., Marques S., y Rodrigues R. (2016). Sobrecarga y calidad de vida del cuidador principal del adulto mayor. Av En Enferm, 34(3). Disponible en: http://www.revistas.unal.edu.co/index.php/avenferm/article/view/58704

Swinkels, J., Tilburg, T., Verbakel, E., y Groenou, E. (2019) Explicando la brecha de género en la carga de cuidado de los cuidadores socios. The Journals of Gerontology: Serie B, 74 (2), 309-317. Disponible en: $\underline{\text { https: }}$ //doi.org/10.1093/geronb/gbx036

Tartaglini MF., Feldberg, C., Hermida, P., Heisecker, S., Dillon, C., Ofman, D., Nuñez, M., y Somale, V. (2020). Escala de sobrecarga del cuidador de Zarit: análisis de sus propiedades psicométricas en cuidadores familiares residentes en Buenos Aires, Argentina. 12(1), 27-35. Disponible en: https://www.elsevier.es/es-revistaneurologia-argentina-301-articulo-escala-sobrecarga-del-cuidador-zarit$\underline{\mathrm{S} 1853002819300862}$

Toro, Y., y Rivas, E. (2016) Cuidadores informales rurales de pacientes dependientes severos. Rev. iberoam. educ. investi. Enferm, 6(1):56-62. Disponible en: https://www.enfermeria21.com/revistas/aladefe/articulo/192/cuidadoresinformales-rurales-de-pacientes-dependientes-severos/

Zepeda, P., y Muñoz, CL. (2019). Sobrecarga en cuidadores principales de adultos mayores con dependencia severa en atención primaria de salud. Gerokomos, 30(1):2-5. [citado 24 de octubre de 2021]. Disponible en: https://scielo.isciii.es/scielo.php?script=sci_arttext\&pid=S1134$\underline{928 \times 2019000100002}$ 